\title{
An improved islanding detection algorithm based on AFDPF
}

\author{
Ma Lulu, ${ }^{1, *}$, Guo Xiujuan ${ }^{1}$, and Wei Liming ${ }^{1}$ \\ ${ }^{1}$ School of Electrical and Computer, Jilin Jianzhu University, Changchun 130118
}

\begin{abstract}
Unplanned islanding is a harmful phenomenon that will cause a series of adverse effects on the power grid. Therefore, islanding detection is a necessary feature of a microgrid with distributed power generation. Based on the Active Frequency Drift with Positive Feedback (AFDPF) algorithm, this paper proposes an improved frequency offset method.The method uses the frequency difference between two cycles to determine the direction of the disturbance signal. The value of the third power is superimposed on the point of common coupling (PCC)to improve detection speed.And check the Nondetection zone of this method based on the Qf0 $\times$ Cnom coordinate system. Finally, through MATLAB/simulink simulation, the effectiveness and superiority of the improved frequency shift islanding detection algorithm are verified.
\end{abstract}

\section{Introduction}

In recent years, with the continuous development of new energy, there are more and more photovoltaic gridconnected systems, but they face many challenges while grid-connected. The islanding effect is a basic challenge for microgrids [1].The islanding effect is that in a system with distributed power sources connected to the grid, the grid is out of power due to some reasons, and the gridconnected inverter does not detect the grid power outage and continues to supply power to the load, so that a power company cannot control "Islanding" phenomenon, this phenomenon will not only cause the voltage and frequency deviation at the grid connection point, but also damage the electrical equipment, and more seriously threaten the personal safety of maintenance personal .Therefore, it is very important to detect islands in time and avoid the harm caused by them. At present, the basic island detection method is mainly divided into two categories: passive detection method and active detection method [2]. Passive detection method is to measure the significant change of the electrical quantity at the point of common coupling (PCC) when the power grid is disconnected to determine whether the islanding effect occurs[3]. The principle of this method is simple, but the detection blind zone is large. The active detection method refers to the introduction of a certain regular disturbance signal to the system by controlling the photovoltaic inverter, and judging whether the islanding occurs according to the change of the response parameter. This method can effectively reduce the detection blind zone. This article optimizes on the basis of the active detection AFDPF algorithm to increase the island detection rate and reduce the blind zone.

\section{Improved algorithm based on AFDPF}

\subsection{AFDPF detection algorithm}

The AFDPF detection method is an improved method of active frequency shifting (AFD). It uses the frequency of the inverter output current as a disturbance signal, and adds positive feedback to the active frequency shift algorithm to overcome the limitations of the AFD method[4]. Fig.1. shows the waveforms of output voltage and current after adding a disturbance signal, where tz is the current dead time.

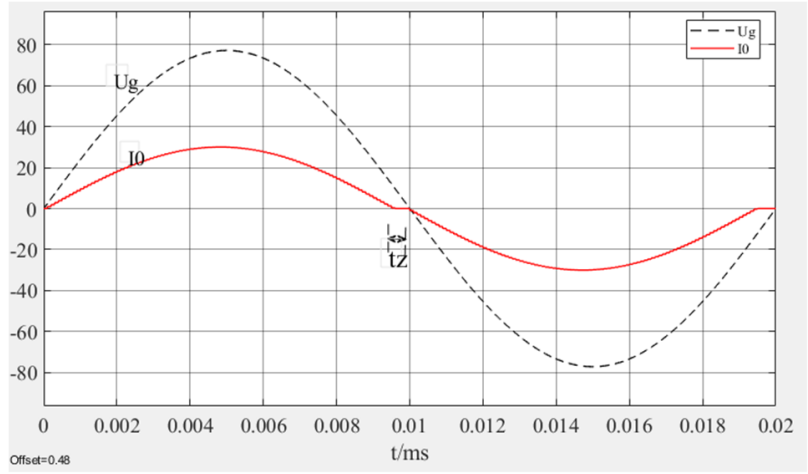

Fig.1. The waveforms of output voltage and current

In the AFD algorithm, the truncation coefficient $\mathrm{cf}$ is defined to represent the frequency disturbance intensity, where the expression of $\mathrm{cf}$ is:

$$
c f=\frac{2 t_{z}}{\mathrm{~T}}
$$

Where $\mathrm{T}$ is the period of the grid voltage.

For the AFD detection algorithm, the larger the cutoff coefficient, the stronger the disturbance added to the system, but this may reduce the power quality of the 
system. In order to increase the detection rate and ensure the power quality, a frequency shift detection method with positive feedback is proposed[5]. Then the expression of AFDPD island detection algorithm is

$$
c f_{n}=\frac{2 t_{z}}{\mathrm{~T}}=c f_{n-1}+\mathrm{k}\left(f_{n}-\mathrm{f}_{\mathrm{g}}\right)
$$

Where $\mathrm{cf}_{\mathrm{n}}$ is the cutoff coefficient of the nth cycle; $\mathrm{cf}_{\mathrm{n}}$ 1 is the cutoff coefficient of the previous cycle; $\mathrm{k}$ is the positive feedback coefficient; $f_{n}$ is the voltage frequency at the PCC point at a certain time; $\mathrm{f}_{\mathrm{g}}$ is the rated frequency of the grid voltage (usually $50 \mathrm{~Hz}$ ) .

The working process of the AFDPF detection algorithm is: when the grid is connected to the grid, the AFDPF islanding detection suppresses the voltage frequency change due to the stability of the grid, and cf remains unchanged; when the islanding phenomenon occurs, the inverter output voltage frequency is disturbed by the disturbance signal cf Due to the effect of positive feedback, the voltage frequency of the inverter in the next cycle has a greater deviation due to the continuous effect of positive feedback. The frequency of the inverter output voltage rises rapidly, and finally exceeds the gridconnected standard to achieve the detection effect.

\subsection{Algorithm improvement}

The analysis of the traditional AFDPF detection algorithm found that the disturbance signal cfn is always disturbed in one direction, and will not change according to the nature of the load. Due to the diversity of loads in the system, it may appear that the direction of the output voltage frequency change of the photovoltaic inverter is opposite to the direction of the introduced disturbance signal, which will result in a slower accumulation of frequency errors, which greatly limits the speed of islanding detection. It will also appear that the load balances the inverter output voltage frequency and cancels the disturbance signal, and the islanding effect cannot be detected, which leads to the disadvantage of a large blind zone in the AFDPF detection algorithm.

In the power system, the characteristics of the load are diverse, roughly divided into inductive, capacitive or resistive. Therefore, international standards such as IEEE1547 and IEEE928 stipulate that the most universal resistance $R$, inductance $L$ and capacitance $C$ are used in parallel to simulate the The load of the system[6]. The overall analysis shows that if the overall load is inductive, the voltage frequency of the common connection point is earlier than the output current frequency. If the overall load is capacitive, the voltage frequency of the common connection point is later than the output current frequency, so it is introduced for different loads. Disturbance signals in different directions can effectively accelerate the deviation of the voltage frequency, thereby speeding up the detection rate, and effectively reducing the detection blind zone.Therefore, a step function is introduced on the basis of the original AFDPF detection algorithm, and the nature of the load is determined by comparing the frequency of the common connection point and the frequency of the grid voltage, thereby determining the direction of the disturbance signal. In order to reduce the superimposition speed of the feedback signal without affecting the direction of the disturbance signal, the cubic term of the feedback frequency shift is added to the original algorithm. The improved algorithm expression is:

$$
\begin{gathered}
c f_{k}=\operatorname{sgn}(\Delta f) \cdot \mathrm{cf}_{0}+\mathrm{k} \Delta f+\mathrm{k}(\Delta f)^{3} \\
\operatorname{sng}(\Delta f)= \begin{cases}1 & f-\mathrm{f}_{\mathrm{g}} \geq 0 \\
-1 & f-\mathrm{f}_{\mathrm{g}}<0\end{cases}
\end{gathered}
$$

The working principle of the improved AFDPF algorithm: When islanding occurs in the microgrid, the voltage frequency of the PCC at the public connection point is compared with the grid frequency. If the voltage frequency of the public connection point is greater than the grid voltage frequency, the introduced disturbance signal $\mathrm{cf}_{\mathrm{n}}>0$ is positive Disturbance, the direction is the same as the direction of load change, accelerate the rise of PCC point voltage frequency, quickly exceed the threshold, the protection device will act and cut off the grid-connected inverter.If the voltage frequency of the common connection point is less than the grid voltage frequency, the introduced disturbance signal $\mathrm{cf}_{\mathrm{n}}<0$ is a reverse disturbance, which is consistent with the direction of the load voltage frequency change, which can accelerate the voltage frequency drop at the PCC point, and quickly fall below the minimum frequency threshold, speeding up detection speed.The improved AFDPF detection algorithm, when islanding occurs, the output voltage frequency of the load inverter of any nature will change significantly, thereby eliminating the defect of the traditional AFDPF islanding detection algorithm of fixed direction frequency disturbance.Moreover, the traditional algorithm also superimposes the third term of the feedback frequency shift, which not only does not change the direction of the disturbance signal, but also accelerates the rise or fall of the voltage frequency of the PCC point, and improves the system islanding detection rate. Fig.2. is a flowchart of the improved algorithm.

\section{Nondetection zone based on $Q_{\mathrm{f} 0} \times \mathrm{C}_{\mathrm{nom}}$ coordinate system}

Although the AFDPF detection algorithm solves the shortcomings of the slow detection speed and large blind area of the AFD algorithm, it still affects the power quality of the system, and the disturbance signal of AFDPF is always in one direction. Due to the variability of the load, when the frequency satisfies the formula (5) Formula, the load phase angle exactly offsets the voltage frequency offset angle, the system will not be able to detect islanding.

$$
\arctan \left[\mathrm{R}\left(2 \pi f \mathrm{C}-\frac{1}{2 \pi f \mathrm{~L}}\right)\right]=\theta_{A F D P F}
$$

In Ref [7] proposed A new method of detection nondetection zone (NDZ) which describe NDZ in the

load quality factor and standardized capacitance coordinate interval $\left(\mathrm{Q}_{\mathrm{fo}} \times \mathrm{C}_{\mathrm{nom}}\right)$, this method can effectively describe the impact of the load on the range of the NDZ, and is widely used. From the Ref [7] and 


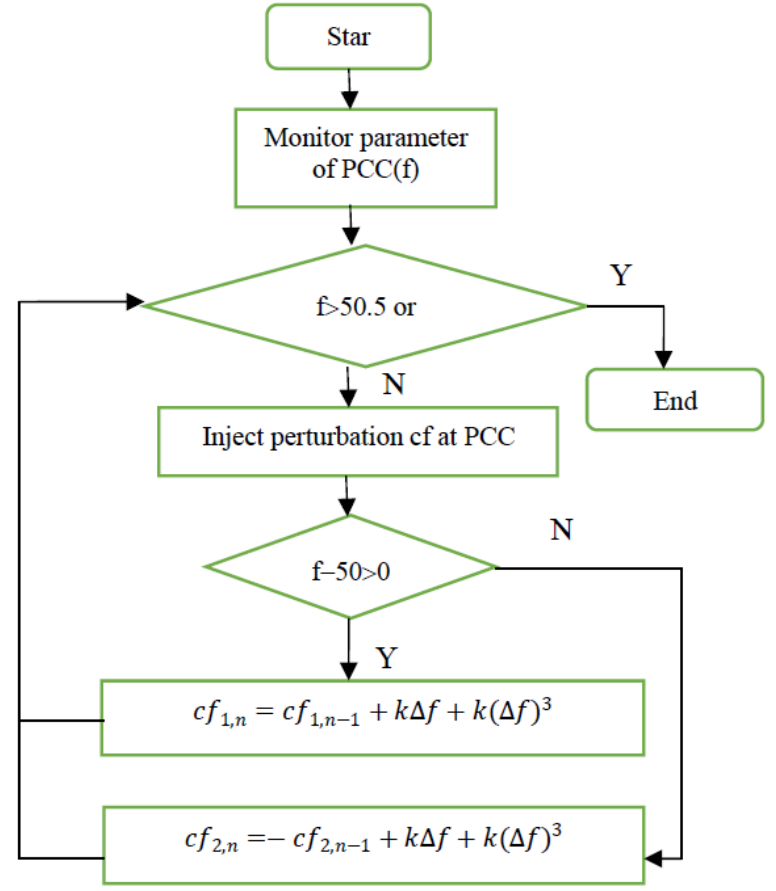

Fig.2. Flowchart of the improved algorithm.

formula(5), it can be seen that the expression of the AFDPF detection algorithm in the $\mathrm{Q}_{\mathrm{fo}} \times \mathrm{C}_{\text {nom }}$ coordinate system is

$$
C_{n o m}=\frac{\tan \frac{\pi\left(c f_{0}-k \Delta f\right)}{2}}{Q_{f 0}}-\frac{\Delta f}{\mathrm{f}_{0}}+1
$$

According to Chinese standards, the voltage frequency can fluctuate up and down $0.5 \mathrm{~Hz}$.Fig.3.shows the NDZ when the feedback coefficient is different when the value of $\mathrm{cf}_{0}$ is 0.02 and 0 .
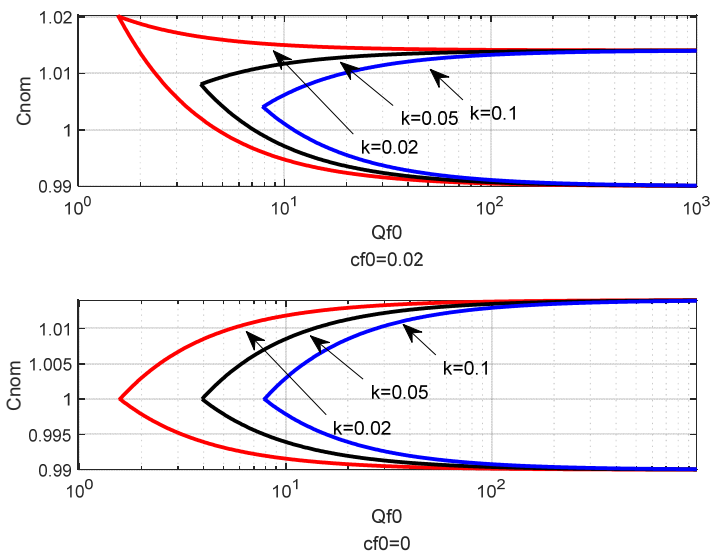

Fig.3. NDZ of AFDPF

According to formulas (3), (4) and (6), it can be seen that the blind zone detection coordinate range of the improved island detection algorithm is:

When the local load is inductive:

$\frac{\tan \frac{\pi}{2}\left(c f_{0}+0.5 k+(0.5 k)^{3}\right)}{Q_{f 0}}+0.98<C_{n o m}<\frac{\tan \left(\frac{\pi}{2} c f_{0}\right)}{Q_{f 0}}+1$

When the local load is capacitive: $\frac{\tan \left(-\frac{\pi}{2} c f_{0}\right)}{Q_{f 0}}+1<C_{n o m}<\frac{\tan \frac{\pi}{2}\left(-c f_{0}-0.5 k-(0.5 k)^{3}\right)}{Q_{f 0}}+1.02(8)$

Fig.4.shows the NDZ of the improved detection algorithm.

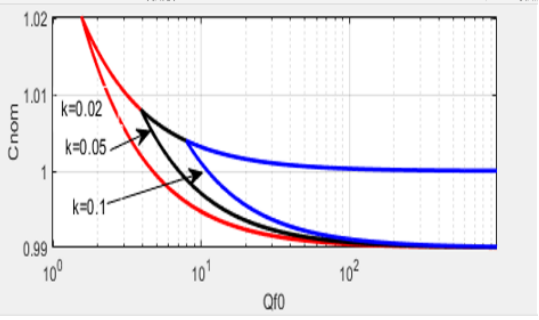

a) The local load is inductive

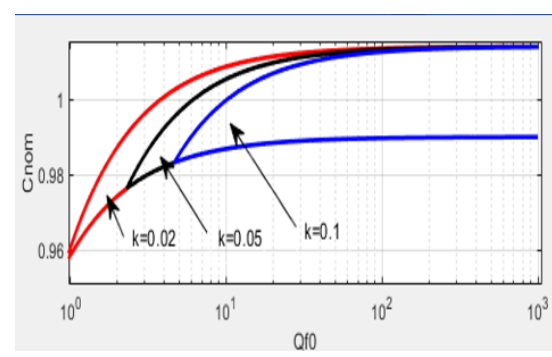

b) The local load is capacitive

Fig.4. The NDZ of improved detection algorithm.

A comparative analysis of Fig. 3 and Fig. 4 shows that the larger the cf0, the smaller the blind zone range, and the better the detection effect; the larger the feedback coefficient $\mathrm{k}$ is, the spatial position of the blind zone moves back. The high quality factor can achieve no NDZ; the improved AFDPF algorithm, regardless of whether the load is inductive or capacitive, NDZ is reduced compared with the traditional algorithm, and it is more usable when performing islanding detection, which improves the accuracy of islanding detection.

\section{Matlab simulation verification}

Build a single-phase photovoltaic grid-connected system model in MATLAB/Simulink. DC source is used to simulate photovoltaic cells, a single-phase full-bridge inverter circuit is used to convert the DC source into an AC source, an L-C filter circuit is used to reduce the harmonic pollution of the system, and an RLC parallel circuit is used to simulate the local load in the gridconnected system. The voltage signal of the PCC point is collected by the grid-connected system island detection module, then uses the phase-locked loop (PLL) to collect the voltage frequency of the common connection point (PCC), and performs the corresponding frequency shift processing on the voltage frequency through the $\mathrm{S}$ Function function.

In order to verify the effectiveness of the improved ADFPF algorithm, take a $2 \mathrm{~kW}$ photovoltaic power generation system as an example, set load resistance $\mathrm{R}=24.2 \Omega$, load quality factor $\mathrm{Q}_{\mathrm{f} 0}$ to 2.5023 , then load resonance frequency is $313.999 \mathrm{~Hz}$, and set the circuit breaker at $0.2 \mathrm{~s}$ Turn off, that is, the grid is powered off for $0.2 \mathrm{~s}$, and the detection algorithm sets the voltage 
frequency protection threshold to $49.5 \sim 50.5 \mathrm{~Hz}$. Figures 4 and 5 show that the blind zone is the smallest when $\mathrm{k}$ is 0.1 and $\mathrm{cf}_{0}$ is 0.02 . Therefore, $\mathrm{k}$ is 0.1 and $\mathrm{cf}_{0}$ is 0.02 during verification. Fig.5.shows the island detection diagram of the traditional AFDPF algorithm and the improved AFDPF algorithm.

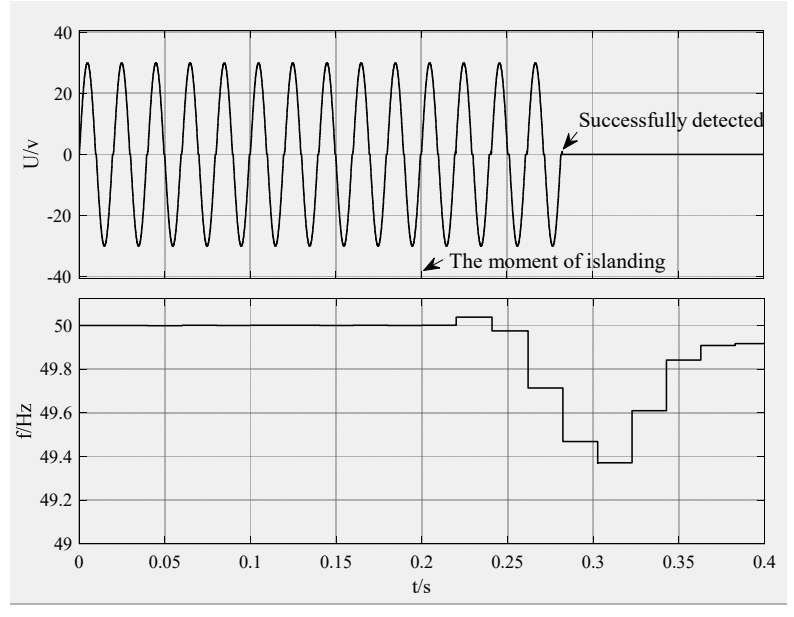

a) Simulation waveform of traditional AFDPF algorithm

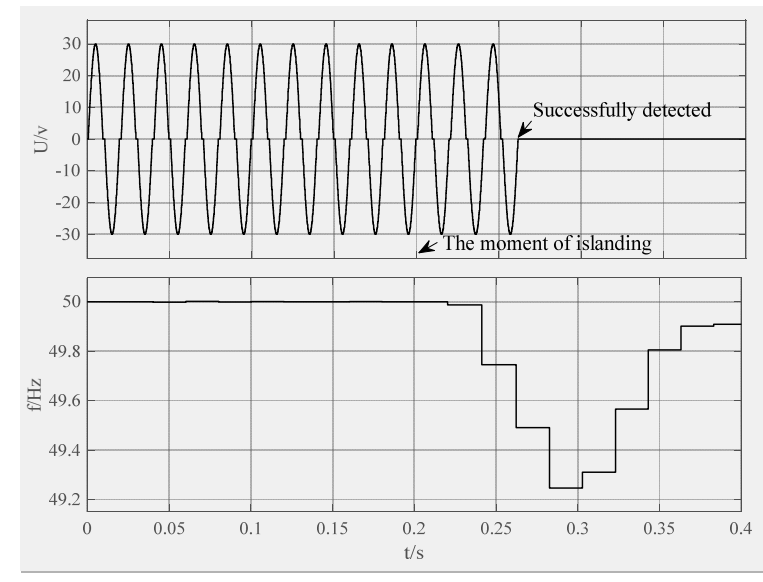

b) Simulation waveform of improved detection algorithm.

Fig.5. Traditional and improved AFDPF algorithm detection diagram

In Fig 5, it can be seen that for the traditional AFDPF islanding detection algorithm, the power grid is cut off at $0.2 \mathrm{~s}$, and after 4 power frequency cycles after the grid is disconnected, the islanding phenomenon is detected in about $0.08 \mathrm{~s}$. The output current of the converter becomes zero, and the photovoltaic system is immediately disconnected from the grid, and the islanding phenomenon is successfully detected. The improved islanding detection algorithm has successfully detected islanding in about $0.06 \mathrm{~s}$ after 3 power frequency cycles after the power grid is disconnected, and the detection time is reduced by one power frequency cycles compared with the traditional detection algorithm. And it can be seen that the improved algorithm has a fast frequency shift and can quickly exceed the threshold to trigger the protection device by the change of voltage frequency. It is verified by simulation that the improved detection algorithm can quickly detect the islanding effect, and make protection in time, and the inverter stops working.
By changing the nature of the local load and the value of $\mathrm{C}_{\mathrm{nom}}$, multiple simulations are performed. According to the multiple sets of simulation data in Table 1, it can be seen that for different load characteristics, the detection time is different. In general, the load in the power system is mostly inductive, so whether it is the traditional AFDFP or the improved one, it can detect the inductive load without blind spots , and the detection time of improved is the shortest, but for capacitive loads, the traditional AFDPF has detection blind zones and no islanding phenomenon. The improved algorithm not only accurately detects the islanding phenomenon, but also the time of detected is short, which improves the detection accuracy and speed, and has certain advantages.

Table 1. Simulation results of islanding detection

\begin{tabular}{l|cc}
\hline Load parameter & $\begin{array}{c}\text { Traditional } \\
\text { algorithm } \\
\text { detection time }\end{array}$ & $\begin{array}{c}\text { Improved } \\
\text { algorithm } \\
\text { detection time }\end{array}$ \\
\hline Pure resistance: & 4 cycles & 3 cycles \\
$\mathrm{R}=11.6 \Omega$ & \\
Inductive load: & & \\
$\mathrm{R}=11.6 \Omega$ & & \\
$\mathrm{L}=14.8 \mathrm{mH}$ & & \\
$\mathrm{C}=600.6 \mu \mathrm{F}$ & 3 cycles \\
$\mathrm{C}$ nom $=0.8773$ & & \\
$\mathrm{Capacitive} \mathrm{load:}$ & & \\
$\mathrm{R}=11.6 . \Omega$ & & \\
$\mathrm{L}=14.8 \mathrm{mH}$ & & \\
$\mathrm{C}=648.6 \mu \mathrm{F}$ & Failed & \\
$\mathrm{C}$ nom $=1.0$ & & \\
\hline
\end{tabular}

\section{Conclusion}

The traditional AFDPF islanding detection algorithm disturbs the singleness of the signal direction and the dependence on the load, which leads to the defects of slow detection speed and large blind area. This paper proposes an improved frequency shift detection method, the disturbance signal can change direction with the change of the load nature, which can effectively solve the defects of the traditional algorithm. Finally, the simulation verifies the superiority and accuracy of the improved algorithm. This method not only speeds up the accumulation speed of voltage frequency error, thereby greatly improving the detection speed, but also effectively reduces the blind zone range.

\section{References}

1. B. Yu, M. Matsui, G. Y u, A review of current antiislanding methods for photovoltaic power system, Sol. Energy 84 (5) (2010) 745-754.

2. M.E. Ropp, M. Begovic, A. Rohatgi, Analysis and performance assessment of the active frequency drift method of islanding prevention, IEEE Trans. Energy Convers. 14 (3) (1999)810-816 
3. L.A.C. Lopes, H. Sun, Performance assessment of active frequency drifting islanding detection methods, IEEE Trans. Energy Convers. 21 (1) (2006) 171-180.

4. D.M. Francesco, L. Marco, D.A. Antonio, Overview of anti-islanding algorithms for PVsystems. Power Electronics and Motion Control Conf.2006 EPEPEMC 2006, Portoroz, Slovenia, 30 Auguste1 September 2006, 2006,pp. 1884-1889.

5. D. Reigosa, F. Briz, C.B. Charro, et al., Active islanding detection using high-frequency signal injection, IEEE Trans. Ind. Electron. 48 (5) (2012) 1588-1597.

6. W.J. Chiang, H.L. Jou, J.C. Wu, Active islanding detection method for inverter-based distribution generation power system, Int. J. Electr. Power Energy Syst. 42 (1) (2012)158-166.

7. D. V elasco, C. Trujillo, G. Garcera, et al., An active anti-islanding method based on phase-PLL perturbation, IEEE Trans. Power Electron. 26 (4) (2011) 1056e1066. 